\title{
Production of iridium metal thin films for application as electrodes in DRAMs and FRAMs
}

\author{
Sakine Shirvaliloo, $^{1,3}$, , Hale Kangarloo ${ }^{2}$ \\ ${ }^{1}$ Department of Medical Physics, Iran University of Medical Sciences, Tehran, Iran \\ ${ }^{2}$ Faculty of Science, IAV, Branch Urmia, Urmia, Iran \\ ${ }^{3}$ Young Researchers Club, Mahabad, Iran \\ Email address: \\ Sakine.shirvaliloo@gmail.com (S. Shirvaliloo), h.kangarloo@yahoo.com (H. Kangarloo)
}

\section{To cite this article:}

Sakine Shirvaliloo, Hale Kangarloo. Production of Iridium Metal Thin Films for Application as Electrodes in DRAMs and FRAMs. Journal of Electrical and Electronic Engineering. Special Issue: Research and Practices in Electrical and Electronic Engineering in Developing Countries. Vol. 3, No. 2-1, 2015, pp. 35-38. doi: 10.11648/j.jeee.s.2015030201.18

\begin{abstract}
Thin films of Noble metals such as Iridium have several potential applications in ICs. Electronic devices are fabricated on the integrated circuits that include transistor, capacitor and resistance. They can be used as electrodes in DRAMs and FRAMs, and as gate electrodes in MOSFETs. Noble metals are excellent metals for electrode fabrication because chemical stability, highly electrical resistance, highly work function and many of them can withstand highly oxidizing conditions. In this paper, emphasis is on reaction mechanisms and limits, leakage currents, electrodes and electrode interfaces and deposition techniques.
\end{abstract}

Keywords: Atomic Layer Deposition, Precursor, Gate Electrode, Dynamic and Ferroelectric Memories, Capacitors

\section{Introduction}

Atomic layer deposition (ALD), also called atomic layer epitaxy (ALE), is a thin film deposition technique developed in Finland in the mid-1970s. Initially, ALD was developed for deposition of materials used in electroluminescent displays but during the years, the selection of materials and their potential applications has expanded [1]. Iridium is chemically very stable, and can withstand highly oxidizing conditions [2]. Therefore, this metal is applicable to ICs as electrodes in dynamic random access memories (DRAMs) and ferroelectric random access memories (FRAMs). Other applications in ICs include gate electrodes in MOSFETs, and seed and barrier layers for copper in interconnect metallization. In addition to ICs, noble metals have applications for example in catalysis, sensors, and magnetic data storage [3-5].

\section{Data and Material}

In this work, we used Iridium thin films. Iridium is the chemical element with atomic number 77 , and is represented by the symbol Ir. Iridium is a very hard, brittle, silvery-white transition metal of the platinum family. Iridium is noble metal similar to silver and gold, and the Platinum group metals ruthenium, rhodium, palladium, osmium, and platinum [2]. Noble metals are called noble because of their chemical stability; they have Positive standard reduction potentials, and many of them are highly resistant to oxidation, even at elevated temperatures [3].

\section{Research Methodology}

In our research study, we have used atomic layer deposition. ALD is a gas phase thin film deposition method which can be regarded as a special modification of the more widely used chemical vapor deposition (CVD) technique [1]. In ALD, The precursors are led into the reaction in alternate pulses. While in CVD, precursors are led into the reaction chamber simultaneously ALD processes for iridium have been reported so far only for $\operatorname{Ir}(\mathrm{acac})_{3}$ and oxygen, and For $\operatorname{Ir}(\mathrm{acac})_{3}$ and hydrogen. Table1 presents a summary of these iridium ALD processes [5].

$\operatorname{Ir}(\mathrm{acac})_{3}$ is solid at room temperature and has a relatively low vapor pressure, 195 which, however, is not a problem in ALD. In the oxygen-based process, $\operatorname{Ir}(\text { acac })_{3}$ was sublimated at $150{ }^{\circ} \mathrm{C}$. Air with a flow rate of $5-40 \mathrm{sccm}$ or pure oxygen with a flow rate of 5-20 sccm was used as the oxygen source [2]. The film growth took place on $\mathrm{Al}_{2} \mathrm{O}_{3}$ surface at 
temperatures of $225^{\circ} \mathrm{C}$ and higher. At $400^{\circ} \mathrm{C}$, signs of thermal self-decomposition of $\operatorname{Ir}(\mathrm{acac})_{3}$ were observed, and therefore, $375{ }^{\circ} \mathrm{C}$ was considered the upper temperature limit for the process. The temperature range for the film growth is in good accordance with the reported thermal stability of $\operatorname{Ir}(\mathrm{acac})_{3}$ in vacuum and in oxygen atmosphere.

Table 1. Summary of the reported iridium ALD processes.

\begin{tabular}{|c|c|c|c|c|}
\hline Metal precursor & T vaporization $\left({ }^{\circ} \mathrm{C}\right)$ & Reaction gas & Flow rate (sccm) & T growth $\left({ }^{\circ} \mathrm{C}\right)$ \\
\hline \multirow{3}{*}{$\operatorname{Ir}(\text { acac })_{3}$} & 150 & Air & $5-40$ & $225-400$ \\
\hline & 150 & $\mathrm{O}_{2}$ & $5-20$ & $225-300$ \\
\hline & N/A & $\mathrm{H}_{2}$ & $\begin{array}{l}\text { separate } \\
\text { reduction step }\end{array}$ & $\begin{array}{l}200-250 / \\
275-450\end{array}$ \\
\hline
\end{tabular}

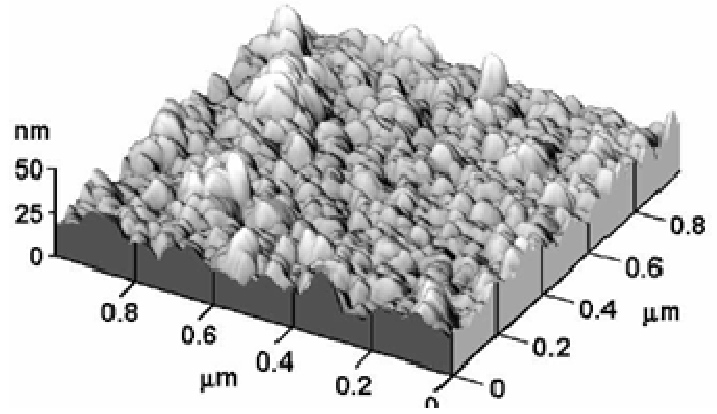

Figure 1. AFM image of a 85-nm iridium film grown at $350^{\circ} \mathrm{C}$ from $\operatorname{Ir}(\text { acac })_{3}$ and air.

Resistivities of about 70-nm thick iridium films grown from $\operatorname{Ir}(\mathrm{acac})_{3}$ and air at temperatures between 225 and $400{ }^{\circ} \mathrm{C}$ were lower than $12 \mu \Omega \cdot \mathrm{cm}$. The film resistivity decreased with increasing growth temperature which was most probably due to increased crystallinity of the films. At $400{ }^{\circ} \mathrm{C}$, there was a small increase in the resistivity, possibly due to the increased impurity contents of the film. The increase in the resistivity with decreasing film thickness is related to an increased effect of grain boundary and surface scattering [2].

The iridium films grown from $\operatorname{Ir}(\mathrm{acac})_{3}$ and oxygen at temperatures of $225-400{ }^{\circ} \mathrm{C}$ were metallic iridium with a preferred (111) crystal orientation as studied by XRD. The films showed a strong tendency to orient the (111) crystal plane, which has the lowest surface energy, parallel to the surface. Similar tendency was also observed with platinum, which is a fcc metal, too. The iridium films had a preferred (111) crystal orientation even at the lowest growth temperatures, implying high surface mobility of the iridium atoms. Figure 14 shows that the (111) crystal orientation is favorable already at the beginning of the growth of an iridium film at $300{ }^{\circ} \mathrm{C}$; in the XRD pattern measured from a $9-\mathrm{nm}$ film, the intensity ratio of the iridium (111) and (200) reflections is $4.6: 1$ while in a randomly oriented powder, the intensity ratio would be $2: 1.21$.

Iridium has been deposited by ALD from $\operatorname{Ir}(\text { acac })_{3}$ and hydrogen on large surface area $\mathrm{Al}_{2} \mathrm{O}_{3}$ and $\mathrm{SiO}_{2}-\mathrm{Al}_{2} \mathrm{O}_{3}$ supports for catalytic applications. The reaction temperature during $\mathrm{Ir}(\mathrm{acac})_{3}$ exposure was $200{ }^{\circ} \mathrm{C}$ [2]. The results imply that when $\operatorname{Ir}(\mathrm{acac})_{3}$ adsorbs on the support surface, one of the three acac ligands is released in reaction with the surface hydroxyl groups. The remaining ligands were removed by a separate reduction step at temperature of $275-450{ }^{\circ} \mathrm{C}$.

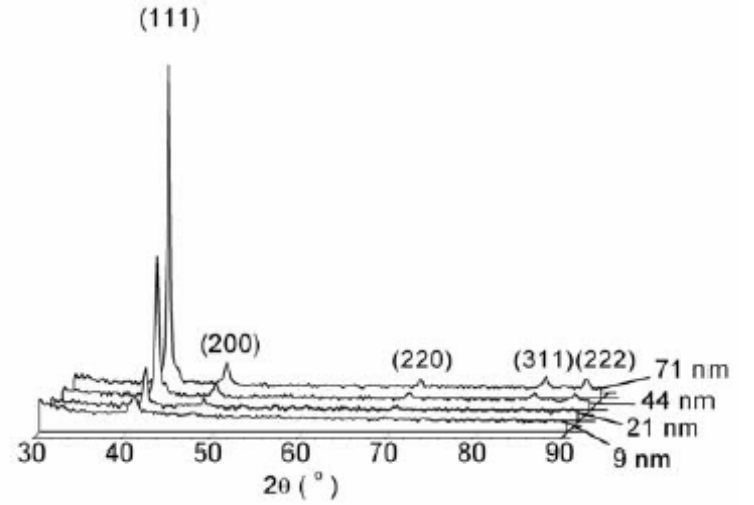

Figure 2. XRD patterns measured from iridium films of different thicknesses. The films were grown at $300^{\circ} \mathrm{C}$.

\section{Results and Analysis}

\subsection{Dynamic Random Access Memory Electrodes}

The data storage in DRAMs is based on introducing a charge into the memory capacitor. The charge must be repeatedly refreshed (thereof the term 'dynamic'), the time interval between the refreshments depending on the leakage current through the capacitor. The charge storage capacity of the capacitor is determined by its capacitance, which can be increased by decreasing the thickness of the dielectric layer, by using a dielectric material with a higher dielectric constant, or by increasing the active capacitor area [6]. Three-dimensional structures provide larger capacitor areas even if the lateral dimensions of the capacitors decrease, and therefore, three-dimensional structures are needed in order to further increase the number of capacitors per unit area, and thus, the storage density of the DRAMs. In stacked DRAM memory cells, the capacitors are fabricated above the silicon substrate. Examples of stacked capacitor structures are shown in Figure [5].
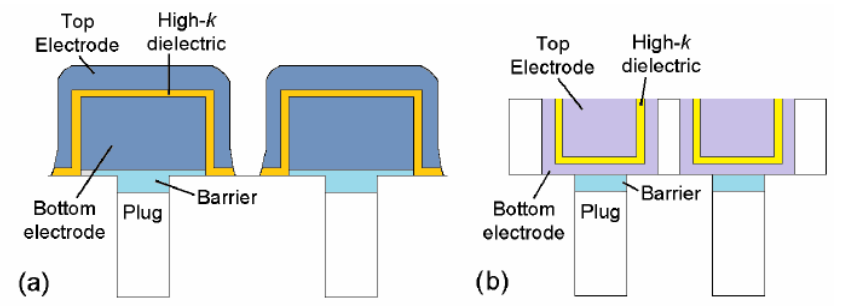

Figure 3. (a) An example of a simple stacked DRAM capacitor structure, and (b) an example of a concave stacked DRAM capacitor structure. 
Noble metals are suitable electrode materials because they remain conductive in highly oxidizing conditions; platinum is highly resistant to oxidation, whereas iridium and ruthenium form conductive oxides $\left(\mathrm{IrO}_{2}\right.$ and $\left.\mathrm{RuO}_{2}\right)$ when they become oxidized. Furthermore, noble metals have high work functions, and thus high barrier for leakage by Schottky emission, that is, thermionic emission in the presence of an applied electric field. The bottom electrode affects the dielectric and electrical properties of the high-k oxide, and plays therefore a significant role in the performance of the memory capacitor. The following sections summarize the noble metal electrode structures studied for DRAM capacitors with $\mathrm{Ta}_{2} \mathrm{O}_{5}$ and BST as the dielectric materials [6].

\subsubsection{Electrodes for $(\mathrm{Ba}, \mathrm{Sr}) \mathrm{TiO}_{3}$}

Iridium is a promising electrode material for BST because good dielectric properties have been reported for BST films grown on iridium electrodes. BST films having a high dielectric constant have been obtained on iridium electrodes. The top electrode material affects the dielectric constant of the BST film as well; higher dielectric constants have been obtained when iridium is used as the top electrode instead of platinum. This difference was explained by a large compressive stress in the BST film induced by the iridium top electrode. The leakage currents reported for BST films grown on iridium are, however, higher than for BST films grown on platinum. High-temperature post-annealing in $\mathrm{O}_{2}$ atmosphere has been reported to lower the leakage currents of BST films grown on iridium electrodes [5].

An iridium oxide layer forms on the surface of the iridium electrode during BST processing at temperatures of $600^{\circ} \mathrm{C}$ or higher. This iridium oxide layer can act as an oxygen diffusion barrier. Iridium films annealed at $700{ }^{\circ} \mathrm{C}$ in $\mathrm{N}_{2}$ atmosphere have been reported to prevent oxygen diffusion better than non-annealed films.85 this is because the number of grain boundaries reduces during the high temperature annealing. Thus, there are fewer pathways for oxygen diffusion. The stability of iridium in contact with silicon is moderate; iridium reacts with silicon when annealed in $\mathrm{O}_{2}$ atmosphere at $650{ }^{\circ} \mathrm{C}$ but is not completely consumed in the reaction with silicon [7].

\subsubsection{Electrodes for $\mathrm{Ta}_{2} \mathrm{O}_{5}$}

Iridium is a potential bottom electrode material for capacitors with $\mathrm{Ta}_{2} \mathrm{O}_{5}$ as the dielectric because high dielectric constants and low leakage currents have been reported for $\mathrm{Ta}_{2} \mathrm{O}_{5}$ grown on ruthenium electrodes. For crystalline $\mathrm{Ta}_{2} \mathrm{O}_{5}$ films, dielectric constants in the range of 45-110 have been reported whereas for amorphous films, the dielectric constant is about 25-32. Thus, in order to obtain higher dielectric constants, the $\mathrm{Ta}_{2} \mathrm{O}_{5}$ films need to be crystallized. The crystallization is usually performed by post-annealing at a temperature of $700{ }^{\circ} \mathrm{C}$ or higher. If the high-temperature annealing is performed in an $\mathrm{O}_{2}$ atmosphere, the ruthenium bottom electrode is likely to become oxidized to ruthenium oxide [2]. The $\mathrm{RuO}_{2}$ formation causes roughening of the $\mathrm{Ta}_{2} \mathrm{O}_{5}$-electrode interface which leads to higher leakage currents. Therefore, in order to prevent ruthenium oxidation, the high-temperature annealing of $\mathrm{Ta}_{2} \mathrm{O}_{5}$ is usually performed in a $\mathrm{N}_{2}$ atmosphere or in vacuum. The dielectric properties of $\mathrm{Ta}_{2} \mathrm{O}_{5}$ can also be improved by $\mathrm{O}_{2}$ or $\mathrm{N}_{2} \mathrm{O}$ plasma treatments or $\mathrm{O}_{2}$ annealing performed at lower temperatures of $350-550{ }^{\circ} \mathrm{C}$ at which oxidation of the ruthenium bottom electrode does not take place.

\subsection{Ferroelectric Random Access Memory Electrodes}

In FRAMs, the data is stored as the polarization state of the ferroelectric film. Switching between two stable polarization states is realized by applying an electric field across the ferroelectric film. FRAMs have two different types of operation principles; the data can be stored in ferroelectric capacitors or in ferroelectric transistors. Ferroelectric capacitors are otherwise similar to DRAM capacitors (Figure3) but the high-k dielectric is replaced by the ferroelectric film. In ferroelectric capacitors, an excess charge is drawn from the circuit and stored to the capacitor if the polarization state of the ferroelectric film is changed during the read-out process. Thus, the polarization state of the ferroelectric film can be determined by detecting the presence or the absence of the switching charge pulse. The magnitude of the switching charge can be increased by using three-dimensional capacitor $\begin{array}{llll}\text { structures [8]. The metal } & \text { The }\end{array}$ -ferroelectric-insulator-semiconductor (MFIS) FET shown in Figure 4 is an example of a ferroelectric transistor [7].

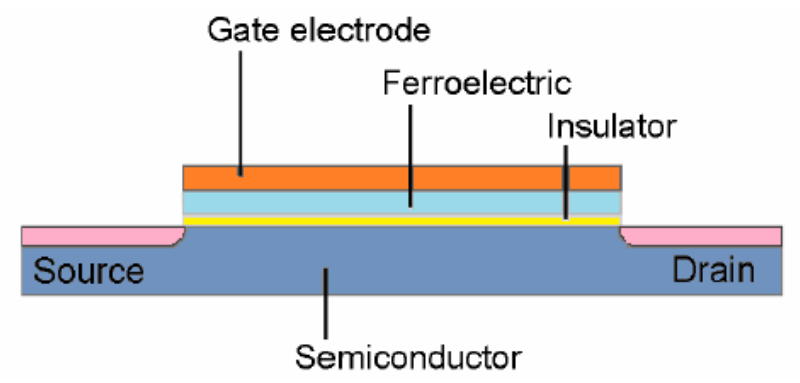

Figure 4. A schematic of a MFIS field effect transistor.

\subsubsection{Electrodes for $\mathrm{Pb}(\mathrm{Zr}, \mathrm{Ti}) \mathrm{O}_{3}$}

Iridium has been shown to be a suitable electrode material for PZT because high remnant polarization, low leakage currents, and good fatigue properties have been obtained for PZT grown on iridium. The good fatigue properties of the PZT films are most probably due to oxidation of the iridium surface during the PZT deposition at temperatures higher than $530{ }^{\circ} \mathrm{C}$. In addition, the $\mathrm{IrOx}$ layer formed on the surface of the iridium electrode prevents diffusion of lead and oxygen through the electrode. Iridium can also be used as an oxygen barrier layer in multilayer electrode structures [8].

\subsubsection{Electrodes for $\mathrm{SrBi}_{2} \mathrm{Ta}_{2} \mathrm{O}_{9}$}

Iridium is a potential electrode material both in ferroelectric capacitors and in ferroelectric transistors having SBT as the ferroelectric. In ferroelectric capacitors, SBT films grown on iridium electrode have been reported to show poor ferroelectric properties, possibly because of oxidation of the iridium surface during SBT processing [8,9]. However, 
iridium and $\mathrm{IrO}_{2}$ can be used as oxygen barrier layers together with platinum electrodes in multilayer electrode structures. Platinum cannot be grown directly on iridium, because platinum and metallic iridium inter diffuse at temperatures higher than $650{ }^{\circ} \mathrm{C}$. Iridium, on the other hand, cannot be grown directly on the poly-Si plug because it forms iridium silicide. In MFMIS transistors, iridium has been used as the lower metal electrode on which the ferroelectric SBT layer is grown. However, bismuth was reported to diffuse into the iridium electrode during high temperature annealing.

\section{Discussion and Conclusion}

The results obtained in this work show that high quality noble metals such as iridium and iridium films can be grown by ALD. All of the processes are based on reactions of the metal precursor with oxygen, the growth temperatures being in the range of $200-450^{\circ} \mathrm{C}$. Iridium films were grown from $\operatorname{Ir}(\mathrm{acac})_{3}$ and oxygen at a temperature range of $225-375^{\circ} \mathrm{C}$. The films were metallic iridium with a preferred (111) crystal orientation. Depending on the thickness, the films had resistivities in the range of $9-18 \mu \Omega \cdot \mathrm{cm}$. The films had smooth surfaces; a $65-\mathrm{nm}$ film grown at $300{ }^{\circ} \mathrm{C}$ had an rms surface roughness of $1.6 \mathrm{~nm}$. At $300{ }^{\circ} \mathrm{C}$, the film growth rate saturated to $0.40 \AA /$ cycle, and the film thickness depended linearly on the number of the growth cycles.

ALD is a suitable method for growing noble metals for the potential applications in ICs which include electrodes in DRAMs and FRAMs, gate electrodes in MOSFETs, and seed layers for copper in interconnect metallization. Noble metal ALD processes taking place in oxidizing conditions can be used when growing DRAM and FRAM top electrodes, gate electrodes for MOSFETs and ferroelectric FETs, and combined seed and barrier layers for copper in interconnect metallization. The DRAM and FRAM bottom electrodes as well as the copper seed layers grown on nitride barriers need to be grown in reducing process conditions.

\section{References}

[1] Ritala, M. and Leskelä, M., Atomic layer deposition of thin films for microelectronics, Electrochem.Soc. Proc., 2003-08 (2003) 479-490.

[2] Aaltonen, T., Ritala, M., and Leskelä, M., Atomic layer deposition of noble metals, in Advanced Metallization Conference 2004 (AMC 2004), (Eds: D. Erb, P. Ramm, K. Masu, and A. Osaki), Materials Research Society (2005) 663-667.

[3] Jylhä, O., Saarinen, R., Lindblad, M., and Krause, O., Iridium on porous supports prepared by ALD for heterogeneous catalysis applications, ALD 2004, Helsinki, August 16-18 (2004).

[4] Aaltonen, T., Ritala, M., Sammelselg, V., and Leskelä, M., Atomic layer deposition of ridium thin films, J. Electrochem. Soc., 151 (2004) G489-G492.

[5] Aaltonen, T., Ritala, M., and Leskelä, M., Atomic layer deposition of noble metals, in Advanced Metallization Conference 2004 (AMC 2004), (Eds: D. Erb, P. Ramm, K. Masu, and A. Osaki), Materials Research Society (2005) 663-667.

[6] Mandelman, J. A., Dennard, R. H., Bronner, G. B., DeBrosse, J. K., Divakaruni, R., Li, Y., and Radens, C. J., Challenges and future directions for the scaling of dynamic random-access memory (DRAM), IBM. J. Res. Develop., 46 (2002) 187-212.

[7] Iwai, H. and Ohmi, S., Silicon integrated circuit technology from past to future, Micro electron. Reliab. 42 (2002) 465-491.

[8] Ishiwara, H., Recent progress of ferroelectric memories, Int. J. High Speed Electron. Syst., 12 (2002) 315-323.

[9] Yoon, D.-S., Roh, J. S., Baik, H. K., and Lee, S.-M., Future direction for a diffusion barrier in future high-density volatile and nonvolatile memory devices, Crit. Rev. Solid State Mater. Sci., 27 (2002)143-226. 